\title{
COVID-19 ECMO Myths Busted
}

\author{
Amita Sharma ${ }^{1}$ Sandeep Sharan ${ }^{1}$ Poonam Malhotra Kapoor ${ }^{1} \quad$ Ujjwal Kumar Chowdhury²
}

1Department of Cardiac Anaesthesia, CTC, AlIMS, New Delhi, India

${ }^{2}$ Department of CTVS, CTC, AlIMS, New Delhi, India
Address for correspondence Dr. Poonam Malhotra Kapoor, Professor, Department of Cardiac Anaesthesia, CTC, AlIMS, New Delhi, India (e-mail: drpoonamaiims@gmail.com).

\begin{abstract}
Keywords

- COVID-19

- ECMO

- Myths

Literature has proven that COVID-19 patients develop pneumonia. Prognosis of this is poor in case of COVID-19 patients developing the following: low lymphocyte count $(<16 \%)$ which gets lower with active COVID-19 infection, sedentary lifestyle, obesity, and uncontrolled diabetes mellitus with no exercise. A lot of dilemmas and myths in this nascent COVID-19 pandemic period exist regarding the use of ECMO. Perplexities such as do we treat the COVID-19 patients on ECMO, as a pulmonary ARDS pneumonia, and/or do we need high PEEP or moderate PEEP, during ECMO for this ARDS; are some common COVID-19 ECMO myths which this short review aims to cover in a question-answer format.
\end{abstract}

\section{COVID-19 ECMO MYTHS}

MYTH 1: Risk factors for COVID-19 include lymphopenia, neutrophil to lymphocyte ratio of more than 3.5 and ventilator support during ECMO

TRUTH! According to Henry et al, ${ }^{1}$ although ECMO is life-saving, yet a word of caution on use of extracorporeal membrane oxygenation (ECMO) in COVID-19 patients, with decreased lymphocyte counts, has been suggested. Patients with decease lymphocyte counts have increased disease severity and mortality. There is some immunological decrease in lymphocytes, as the ECMO run progresses. ${ }^{2}$

Literature has proven that COVID-19 patients develop pneumonia. Prognosis of this is poor, in case of COVID-19 patients developing the following: low lymphocyte count $(<16 \%)$ which gets lower with active COVID-19 infection; sedentary lifestyle; obesity; and uncontrolled diabetes mellitus (DM) with no exercise. All young people below 20 years had lymphocyte count percentage of approximately $40 \%$; if neutrophil and lymphocyte ratio is seen to be more than 3.5, then these are COVID-19 patients who will definitely require ventilator support and venovenous ECMO to aid in the ventilation. ECMO in its course induces a substantial decrease in the number and function of lymphocytes. ${ }^{3}$
As COVID-19 recovery warrants lymphocyte repletion and not depletion, it is imperative that lymphocyte count should be closely monitored in COVID-19 patients receiving ECMO.

\section{MYTH 2: Mortality is higher on ECMO when COVID-19 patients show higher values of interleukin- 6}

TRUTH! It has been amply shown by Rvanand colleagues that concentration of interleukin differed significantly between survivors and nonsurvivors, with the latter having up to 1.7 to 2 times higher values of IL-6. During ECMO, IL-6 concentration is elevated in both children and adults. These remained high in nonsurvivors and decreased to normal, which on ECMO in those who survived and decreased to normal, while on ECMO in those who survived ECMO. Increased IL-6 levels are associated with parenchyma damage in the lungs of animals kept on venovenous ECMO. Thus, it is important to monitor the immunological status of the patient before putting the patient on ECMO. For good prognosis of COVID-19 and for ECMO to be of benefit to the COVID-19 patient, tracking interleukin-6 levels is as important as tracking the lymphocyte and neutrophil count. $^{4}$ published online

15 June 2020
Dol https://doi.org/

$10.1055 / \mathrm{s}-0040-1713490$ ISSN 2457-0206.
(C)2020 Official Publication of

The Simulation Society (TSS), accredited by International Society of Cardiovascular Ultrasound (ISCU).
License terms

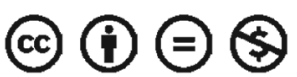




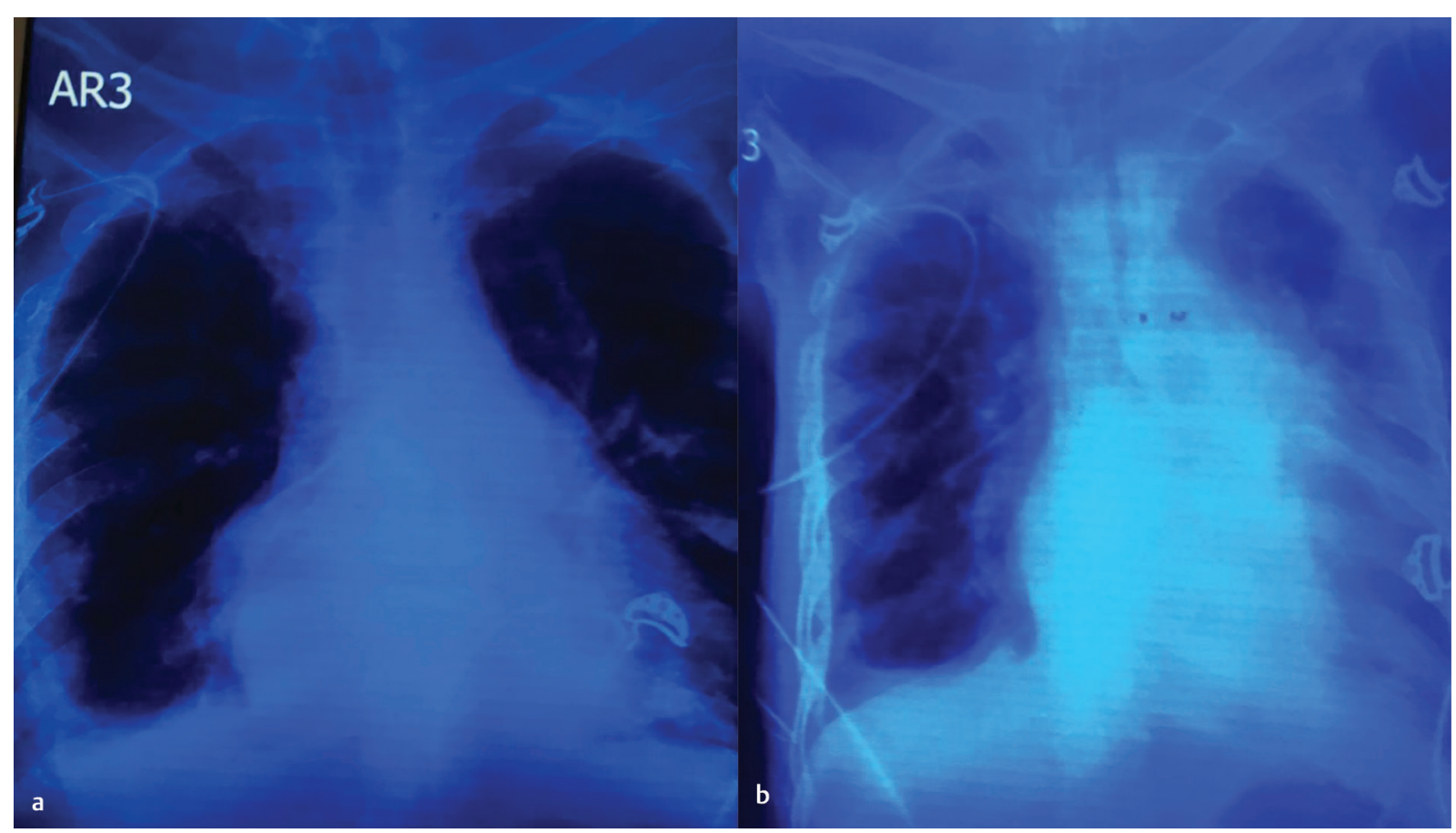

Fig. 1 (a,b) Post-MVR COVID-19 patient, X-ray chest PAV, day 1 and day 10 postoperatively on ECMO weaning. Abbreviations: ECMO, extracorporeal membrane oxygenation; MVR, mitral valve replacement.

\section{MYTH 3: X-ray chests show worsening COVID-19 infection as the Disease progresses in time}

IT IS A MYTH: Ground glass opacities and consolidation are apparent on anteropostero (APV) of chest X-ray from day 1 itself, although the upper respiratory infection (URI) maybe mild. It may progress to new consolidation and infiltrates in the lower lobes as well, as the disease progresses ( $\boldsymbol{- \text { Fig. } \mathbf { 1 } )}$ to pulmonary congestion, due to volume overload during venovenous ECMO run. As ECMO functions with continuous renal replacement therapy (CRRT), pulmonary congestion and consolidation among patients remarkably improve. ${ }^{5}$

\section{MYTH 4: COVID-19 X-ray chest and timing of VV ECMO insertion-VV ECMO can be initialed anytime in COVID-19 infection. The timing of ECMO insertion is immaterial}

IT IS A MYTH: To treat COVID-19 pneumonia with ECMO, it is important to recognize lung plasticity on X-ray chest and start ECMO as per the ELSO guideline. For this, the timing of ECMO introduction must be appropriate. On day 3 of infection, postintubation, most COVID-19 patient's show a respiratory ECMO survival prediction score of point 1 , with an expected survival of $60 \%$. It is in this phase that biomarkers like Krebs vended lungen-6 (KL-6) and surfactant Protein-D (SP-D), which nicely depict lung injury, did not increase. The pneumonia caused by SARS-COVID19, although of slow progression, is severe in intensity. ECMO should be introduced at a time when lung plasticity in patients is maintained. ${ }^{6}$

\section{MYTH 5: Does ECMO attenuate hypoxic pulmonary vasoconstriction (animal studies) during COVID19 infection}

TRUTH! During severe respiratory failure, as in COVID19 infection, hypoxic pulmonary vasoconstriction (HPV) is partly suppressed, but may still play a role in increasing pulmonary vascular resistance (PVR). Experimental studies have suggested that the patients at the time of corona infection are dependent on the pulmonary venous oxygenation, the $\mathrm{PvO}_{2}$. Thus, $\mathrm{PvO}_{2}$ is increased with the use of venovenous ECMO; there would a decrease in pulmonary vascular resistance and thus PV would be alternate respiratory failure.

Holzgraefe et al's animal studies in 2020 have concluded that: ${ }^{7}$

$>$ There is an increase in $\mathrm{PvO}_{2}$ by the use of VV ECMO on PVR, depending on the amount of alveolar hypoxia present. The right ventricular afterload may not always decrease with ECMO when alveolar hypoxia is very severe. ${ }^{8}$

> Pinsky et al have shown in 2006 that hyperinflation increases PVR, and that weaning from mechanical ventilation is a cardiovascular stress.

> The vasomotor tone when increased, and also when lung volume is reduced, gets HPV activated. PEEP and positive 
airways pressure, along with recruitment maneuvers, may reverse HPV and thus reduce pulmonary artery pressures.

\section{MYTH 6: Some COVID-19 patients on ECMO are a sedation nightmare}

TRUTH! Commonly used intravenous (IV) opioids include fentanyl, hydromorphone and morphine, while sedatives often include propofol, dexmedetomidine and benzodiazepines. The choice of agent used is dependent on the desired physiological endpoint, while balancing unwanted adverse effects of each individual agent.

All sedatives like propofol, injecting dexmedetomidine infusion at a dose, keeps the tachycardia in check. The drugs should be individualized, and high deep levels of sedation are need of the hour. ${ }^{9}$

\section{MYTH 7: Powered air-purifying respirators and level} 3 infection control precaution with PPE should be included during ECMO cannulation and maintenance TRUTH! During ECMO cannulation, replacement of oxygenator, other invasive procedures, and level 3 infection control precautions should be exercised including the value of powered air-purifying respirators (PAPR), protective suites, disposable sterile surgical gowns and three-layered sterile gloves. During routine ECMO therapy rounding, "PAPR" is not required. ${ }^{10}$

\section{MYTH 8: Venovenous ECMO is better than venoarterial ECMO in COVID-19 patients}

TRUTH! Venovenous is better than venoarterial! The role and exact utility ECMO for COVID-19 pandemic patients use remains unclear. It is of great utility for aiding ventilation and removing carbon dioxide. ${ }^{11}$

ECMO has been shown in many publications from China to have played a role in the treatment of some patients afflicted with COVID-19, who have the refractory hypoxia patient not responding to aggressive lung protective ventilatory therapy; even the control has provided interim guidelines, wherein, an ECMO should considered according to the state management algorithm for acute respiratory distress syndrome (ARDS) in supporting patients with viral respiratory tract infection. Europe too has used ECMO in many patients of COVID-19 during this pandemic, but the definitive position of ECMO use and trial results are as yet awaited.

As of now, until the heart is involved, venovenous ECMO is better to insert, particularly, via peripheral route. VA ECMO is reserved for patients with respiratory failure and cardiogenic shock. ${ }^{11}$

MYTH 9: ECMO in COVID-19 infection has no role to play if there is acidosis and oxygenation deteriorates despite aggressive, ongoing ventilatory management IT IS A MYTH: As some severe COVID-19 patients deteriorate rapidly, ECMO insertion should not be delayed, if any of the following criteria are seen in a COVID patient with aggressive ventilation. The criteria are as follows:

1. $\mathrm{PaO}_{2} / \mathrm{FiO}_{2}$ ratio $<50 \mathrm{~mm} \mathrm{Hg}$ for more than 1 hour.

2. $\mathrm{PaO}_{2} / \mathrm{FiO}_{2}<80 \mathrm{~mm} \mathrm{Hg}$ for more than 2 hours.

3. Existence of uncompensated respiratory acidosis with $\mathrm{pH}<7.2$ for more than 1 hour.

ECMO in COVID-19 infection has no role to play if there is acidosis and $\mathrm{PaO}_{2} / \mathrm{FiO}_{2}$ ratios deteriorate despite aggressive ventilator. ${ }^{12}$

\section{MYTH 10: Not all patients will improve with ECMO support}

IT IS A MYTH: As is standard with usual ECMO care, clinicians should be continuously evaluating when ECMO no longer provides a positive benefit: risk ratio and should at that point return to conventional management regardless of how long the patient has been on ECMO. During times of limited resources, this becomes especially important and while the definition will be hospital- or region-specific, observing no lung or cardiac recovery after approximately 21 days (these are general guidelines which may not apply to specific COVID-19 patients, depending on local circumstances) on ECMO can be considered futile, and the patient can be returned to conventional management (-Fig. 2).

MYTH 11: Triage with high-concentration of humidified oxygen and low levels of positive endexpiratory pressure as initial therapy before ECMO is not needed in COVID-19 patients

IT IS A MYTH: Before intubating a COVID-19 patient and putting him or her on ECMO, it is important to consider a trial of high-flow nasal oxygen for patients with some hypoxia. This may obtund the need for intubation and mechanical ventilation, as high-humidified oxygen levels and low levels of positive end-expiratory pressure (PEEP) can aid in eliminating carbon dioxide. WHO guidelines support the above but advocate close monitoring of clinical deterioration and emergency mechanical ventilation subsequently. ${ }^{14}$

\section{Conclusion}

A lot of dilemmas and myth in this nascent COVID-19 pandemic period exist regarding the use of ECMO. Perplexities such as do we treat the COVID-19 patients on ECMO, as a pulmonary ARDS pneumonia, and/or do we need high PEEP or moderate PEEP? Do we manage patients with a wet or dry circuit for mucus plugging and secretion management? Are some ongoing dilemmas with ECMO of use in corona patients today. Time will tell definitive tales tomorrow in relation to COVID-19 ECMO myths. 


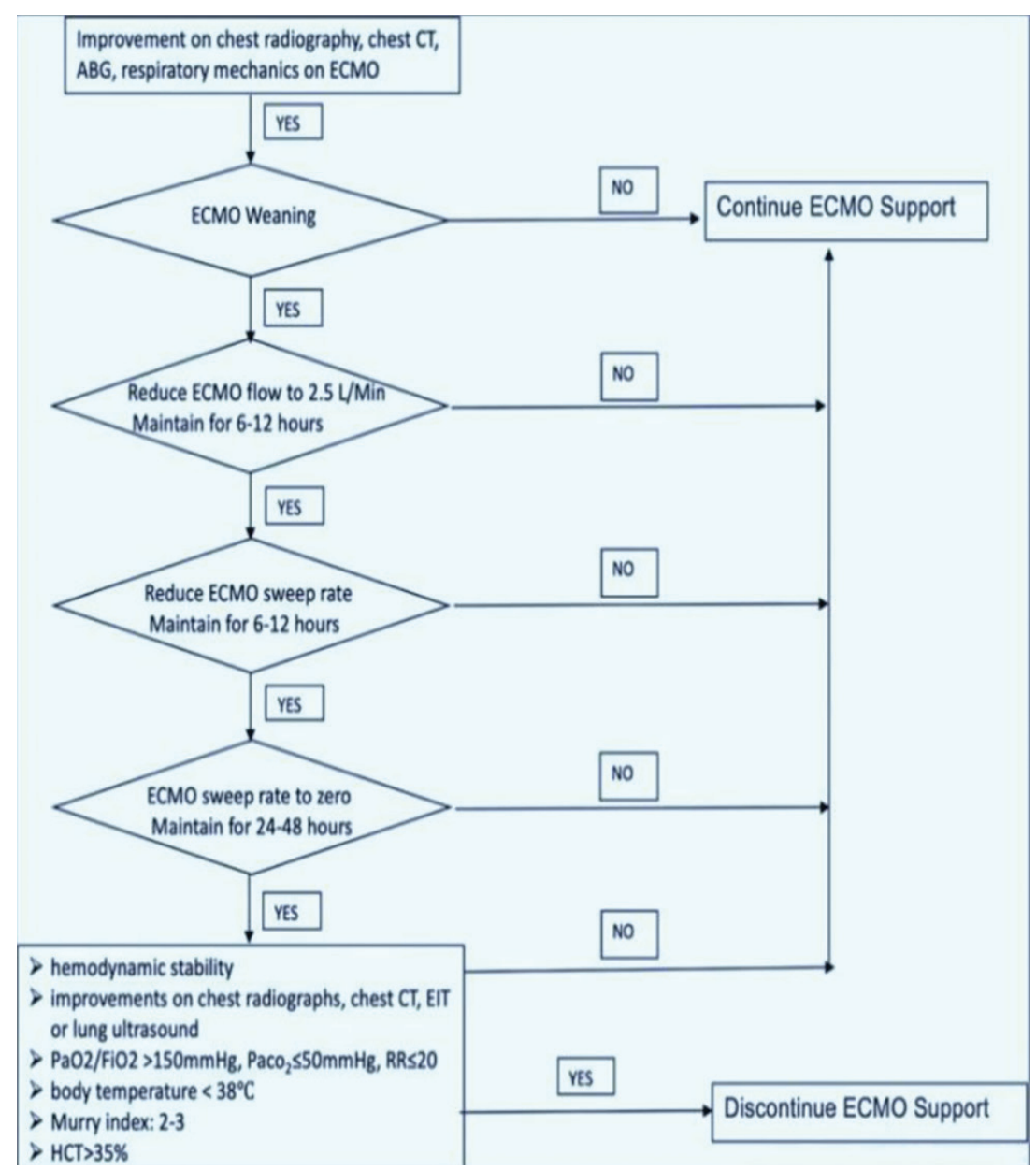

Fig. 2 ECMO weaning protocol in COVID-19 patients. ABG, arterial blood gas; COVID-19, coronavirus disease 2019; CT, computed tomography; ECMO, extracorporeal membrane oxygenation; EIT, electrical impedance tomography; FiO2, fractional of inspired oxygen; HCT, hematocrit; PaCO2, partial pressure of carbon dioxide; $\mathrm{PaO} 2$, partial pressure of oxygen; RR, respiratory rate. ${ }^{13}$

\section{Conflict of Interest}

None.

\section{References}

1 Henry BM. COVID-19, ECMO, and lymphopenia: a word of caution. Lancet Respir Med 2020;8(4):e24

2 Yang $\mathrm{X}, \mathrm{Yu} \mathrm{Y}$, Xu J, et al. Clinical course and outcomes of critically ill patients with SARS-CoV-2 pneumonia in Wuhan, China: a single-centered, retrospective, observational study. Lancet Respir Med 2020;(e-pub ahead of print). doi: 10.1016/ S2213-2600(20)30079-5

3 Ruan Q, Yang K, Wang W, Jiang L, Song J. Clinical predictors of mortality due to COVID-19 based on an analysis of data of 150 patients from Wuhan, China. Intensive Care Med 2020;(e-pub ahead of print). doi: 10.1007/s00134-020-05991-X

4 Bizzarro MJ, Conrad SA, Kaufman DA, Rycus P; Extracorporeal Life Support Organization Task Force on Infections, Extracorporeal Membrane Oxygenation. Infections acquired during extracorporeal membrane oxygenation in neonates, children, and adults. Pediatr Crit Care Med 2011;12(3):277-281
5 Risnes I, Wagner K, Ueland T, Mollnes T, Aukrust P, Svennevig J. Interleukin-6 may predict survival in extracorporeal membrane oxygenation treatment. Perfusion 2008;23(3): 173-178

6 Shi J, Chen Q, Yu W, et al. Continuous renal replacement therapy reduces the systemic and pulmonary inflammation induced by venovenous extracorporeal membrane oxygenation in a porcine model. Artif Organs 2014;38(3):215-223

7 Holzgraefe B, Broomé M, Kalzén H, Konrad D, Palmér K, Frenckner B. Extracorporeal membrane oxygenation for pandemic H1N1 2009 respiratory failure. Minerva Anestesiol 2010;76(12):1043-1051

8 Pinsky MR. The hemodynamic consequences of mechanical ventilation: an evolving story. Intensive Care Med 1997;23(5):493-503

9 Beitler JR, Sarge T, Banner-Goodspeed VM, et al; EPVent-2 Study Group. Effect of titrating positive end-expiratory pressure (PEEP) with an esophageal pressure-guided strategy vs an empirical high PEEP-FiO2 strategy on death and days free from mechanical ventilation among patients with acute respiratory distress syndrome: a randomized clinical trial. JAMA 2019;321(9):846-857 
10 Wiedemann HP, Wheeler AP, Bernard GR, et al; National Heart, Lung, and Blood Institute Acute Respiratory Distress Syndrome (ARDS) Clinical Trials Network. Comparison of two fluid-management strategies in acute lung injury. N Engl J Med 2006;354(24):2564-2575

11 Wang D, Hu B, Hu C, et al. Clinical characteristics of 138 hospitalized patients with 2019 novel coronavirus-infected pneumonia in Wuhan, China. JAMA 2020;(e-pub ahead of print). doi: 10.1001/jama.2020.1585

12 MacLaren G, Fisher D, Brodie D. Preparing for the most critically ill patients with COVID-19: The potential role of extracorporeal membrane oxygenation. JAMA 2020 (e-pub ahead of print). doi: 10.1001/jama.2020.2342

13 Bartlett Robert H., Ogino Mark TBrodie, Daniel, McMullan David M., Lorusso Roberto, et al. Initial ELSO Guidance Document: ECMO for COVID-19 patients with severe cardiopulmonary failure. ASAIO Journal 2020;66(5):472-474

14 Matthay MA, Aldrich JM, Gotts JE. Treatment for severe acute respiratory distress syndrome from COVID-19 Lancet. Respir Med 2020;(e-pub ahead of print). doi: 10.1016/ S2213-2600(20)30127-2 